\title{
Polyominoes determined by permutations
}

\author{
I. Fanti ${ }^{2}$, A. Frosini ${ }^{1}$, E. Grazzini ${ }^{2}$, R. Pinzani ${ }^{2}$ and S. Rinaldi ${ }^{1}$ \\ ${ }^{1}$ Dipartimento di Scienze Matematiche e Informatiche, Pian dei Mantellini, 44, 53100, Siena, Italy. \\ ${ }^{2}$ Dipartimento di Sistemi e Informatica, Viale Morgagni, 65, 50134, Firenze, Italy.
}

In this paper we consider the class of permutominoes, i.e. a special class of polyominoes which are determined by a pair of permutations having the same size. We give a characterization of the permutations associated with convex permutominoes, and then we enumerate various classes of convex permutominoes, including parallelogram, directedconvex, and stack ones.

Keywords: enumeration of combinatorial objects, convex polyominoes, permutations.

\section{Basics on polyominoes}

In the plane $\mathbb{Z} \times \mathbb{Z}$ a cell is a unit square, and a polyomino is a finite connected union of cells having no cut point. Polyominoes are defined up to translations. A column (row) of a polyomino is the intersection between the polyomino and an infinite strip of cells whose centers lie on a vertical (horizontal) line. The enumeration problem for general polyominoes is difficult to solve and still open. The number $a_{n}$ of polyominoes with $n$ cells is known up to $n=56[\mathrm{JG}]$ and asymptotically, this number satisfies the relation $\lim _{n \rightarrow \infty}\left(a_{n}\right)^{1 / n}=\mu, 3.96<\mu<4.64$, where the lower bound is a recent improvement of [BMRR].

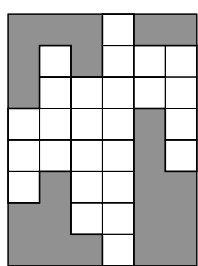

(a)

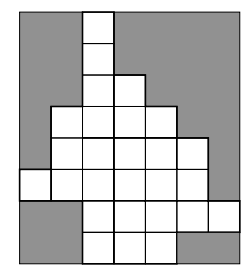

(b)

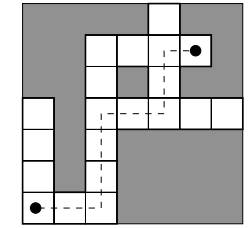

(c)

Fig. 1: (a) column-convex polyomino; $(b)$ a convex polyomino; $(c)$ a directed (not convex) polyomino.

In order to simplify many problems which are still open on the class of polyominoes, several subclasses were defined by combining two notions: the geometrical notion of convexity, and the notion of directed growth, which comes from statistical physics. A polyomino is said to be column-convex [row-convex] when its intersection with any vertical [horizontal] line is convex (Fig. 1 $(a)$ ). A polyomino is convex if it is both column and row convex (Fig. 1 $(b)$ ). In a convex polyomino the semi-perimeter is given by the sum of the number of rows and columns, while the area is the number of its cells.

A polyomino $P$ is said to be directed when every cell of $P$ can be reached from a distinguished cell, called the root (usually the leftmost at the lowest ordinate), by a path which is contained in $P$ and uses only north and east unit steps (Fig. 1 $(c)$ ). Figure $2(d)$ depicts a polyomino that is both directed and convex. Moreover we can define three types of directed and convex polyominoes, i.e. the Ferrers diagrams (Fig. 2 $(a)$ ), the parallelogram polyominoes (Fig. 2 (b)), and the stack polyominoes (Fig.2( $(c)$ ). As Figure 2 shows, each of these three subsets can be characterized, in the set of convex polyominoes, by the fact that two or three vertices of the minimal bounding rectangle of the polyomino must also belong to the polyomino itself.

The number $f_{n}$ of convex polyominoes with semi-perimeter $n \geq 2$ was obtained by Delest and Viennot, in $[\mathrm{DV}]$ :

$$
f_{n+2}=(2 n+11) 4^{n}-4(2 n+1)\left(\begin{array}{c}
2 n \\
n
\end{array}\right), \quad n \geq 0 ; \quad f_{0}=1, \quad f_{1}=2 .
$$

Moreover, it is well known $[\mathbf{S}]$ that the number of parallelogram polyominoes with semi-perimeter $n \geq 2$ is equal to the $(n-1)$ th Catalan number, where Catalan numbers are defined by 


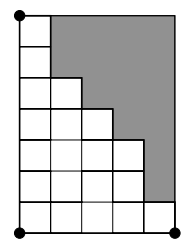

(a)

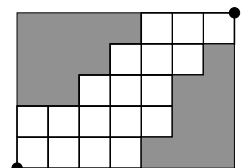

(b)

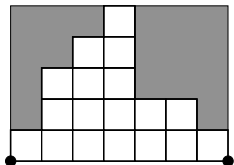

(c)

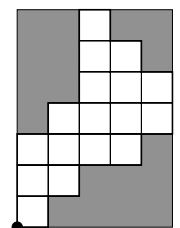

(d)

Fig. 2: (a) A Ferrers diagram; (c) A parallelogram polyomino; (c) A stack polyomino; $(d)$ A directed-convex polyomino.

$$
c_{n}=\frac{1}{n+1}\left(\begin{array}{c}
2 n \\
n
\end{array}\right) .
$$

Finally, the number of directed-convex polyominoes with semi-perimeter $n \geq 2$ is equal to $b_{n-2}$, where $b_{n}$ are the central binomial coefficients,

$$
b_{n}=\left(\begin{array}{c}
2 n \\
n
\end{array}\right) .
$$

\section{Convex permutominoes}

Let $P$ be a polyomino without holes having $n-1$ rows and $n-1$ columns, $n \geq 2$. Without loss of generality, throughout the paper we assume that the south-west edge of the minimal bounding rectangle of the considered polyomino is placed in the point $(1,1)$. Let $A_{1}, B_{1}, \ldots, A_{n}, B_{n}$ be the sequence of the vertices of $P$ obtained by visiting the boundary in clockwise orientation, starting from the lowest point in the leftmost column. We say that $P$ is a permutomino of size $n$ if the sets of points $\mathcal{A}=\left\{A_{1}, \ldots, A_{n}\right\}$ and $\mathcal{B}=\left\{B_{1}, \ldots, B_{n}\right\}$ represent two permutation matrices of size $n$.

Moreover, let us re-arrange the points of $\mathcal{A}$ (resp. $\mathcal{B}$ ) according to the increasing value of their abscissa, giving $\left\{\left(1, y_{1}(A)\right), \ldots,\left(n, y_{n}(A)\right)\right\}$ (resp. $\left\{\left(1, y_{1}(B)\right), \ldots,\left(n, y_{n}(B)\right)\right\}$, and let

$$
\begin{aligned}
& \pi_{1}(P)=\left(y_{1}(A), y_{2}(A), \ldots, y_{n}(A)\right), \\
& \pi_{2}(P)=\left(y_{1}(B), y_{2}(B), \ldots, y_{n}(B)\right) ;
\end{aligned}
$$

we say that the permutomino $P$ is associated with the pair of permutations $\left(\pi_{1}(P), \pi_{2}(P)\right)$ (briefly, $\left(\pi_{1}, \pi_{2}\right)$ ); for simplicity the $i$ th element of $\pi_{1}$ (resp. $\pi_{2}$ ) will be denoted by $\pi_{1}(i)$ (resp. $\pi_{2}(i)$ ). By construction we have that for any permutomino $\pi_{1}(1)<n$ and $\pi_{1}(n)>1$. Figure 3 shows two permutominoes and the associated permutations; we remark that the size of the permutomino, defined as the dimension of the associated permutations, is equal to 5, while the side of the bounding square is 4 .

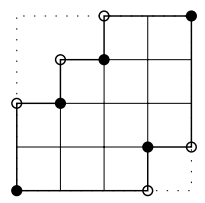

$$
\begin{aligned}
& \pi_{1}=(1,3,4,2,5) \\
& \pi_{2}=(3,4,5,1,2)
\end{aligned}
$$

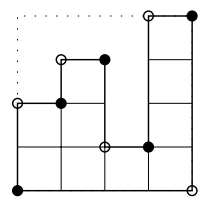

$$
\begin{aligned}
& \pi_{1}=(1,3,4,2,5) \\
& \pi_{2}=(3,4,2,5,1)
\end{aligned}
$$

Fig. 3: Two permutominoes and the associated permutations. The permutation $\pi_{1}$ (resp. $\pi_{2}$ ) is represented by black (resp. white) dots.

In [I] F. Incitti introduced the class of permutominoes while studying the problem of determining the $\widetilde{R}$-polynomials (related with the Kazhdan-Lusztig R-polynomials) associated with a pair $(x, y)$ of permutations. Concerning the class of convex polyominoes, our definition (though different) turns out to be equivalent to Incitti's one, which is more general but uses some algebraic notions not necessary in this paper. 
Here we will focus our interest on the class of convex polyominoes which are also permutominoes (see Figure 4). As a start we consider the problem of giving a characterization of the set of permutations associated with convex permutominoes,

$$
\left\{\left(\pi_{1}(P), \pi_{2}(P)\right): P \text { is a convex permutomino }\right\} .
$$

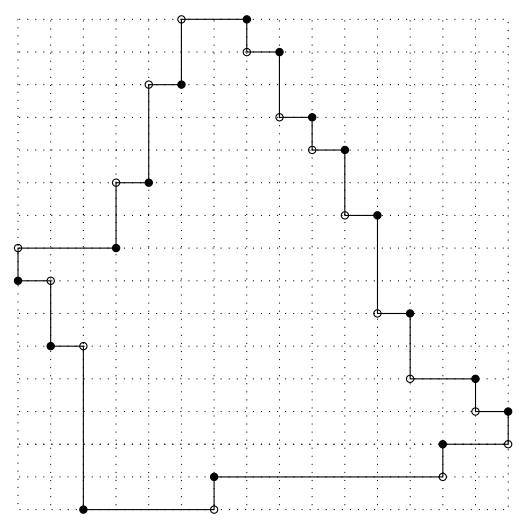

$$
\begin{aligned}
& \pi_{1}=(8,6,1,9,11,14,2,16,15,13,12,10,7,3,5,4) \\
& \pi_{2}=(9,8,6,11,14,16,1,15,13,12,10,7,5,2,4,3)
\end{aligned}
$$

Fig. 4: A convex permutomino and the associated permutations.

In order to do this, we start by observing that convex permutominoes satisfy interesting symmetry properties: let $P$ be a convex permutomino, and let $\left(\pi_{1}(P), \pi_{2}(P)\right)$ be the associated permutations; moreover, let $P_{x}$ (resp. $P_{y}$ ) be the reflection of $P$ with respect to the $x$-axis (resp. $y$-axis). It can easily be proved that:

1. $\pi_{1}\left(P_{x}\right)$ is defined by $\pi_{1}\left(P_{x}\right)(i)=(n+1)-\pi_{2}(P)(i)\left(\right.$ resp. $\left.\pi_{2}\left(P_{x}\right)(i)=(n+1)-\pi_{1}(P)(i)\right)$.

2. $\pi_{1}\left(P_{y}\right)=\pi_{2}(P)^{M}$, where $\pi_{2}^{M}$ denotes the reversal of $\pi_{2}$, and $\pi_{2}\left(P_{y}\right)=\pi_{1}(P)^{M}$.

Thus, let $\mathcal{C}_{n}$ be the set of convex permutominoes of size $n$, and

$$
\widetilde{\mathcal{C}_{n}}=\left\{\pi_{1}(P): P \in \mathcal{C}_{n}\right\}, \quad \widetilde{\mathcal{C}}_{n}^{\prime}=\left\{\pi_{2}(P): P \in \mathcal{C}_{n}\right\}
$$

Trivially we have that for any permutation $\zeta$,

$$
\zeta \in \widetilde{\mathcal{C}_{n}} \quad \text { if and only if } \quad \zeta^{M} \in \widetilde{\mathcal{C}}_{n}^{\prime},
$$

hence $\left|\widetilde{\mathcal{C}}_{n}\right|=\left|\widetilde{\mathcal{C}}_{n}^{\prime}\right|$, for all $n \geq 2$. For instance, the reader can check that there are 4 convex permutominoes of size 3 (i.e. semi-perimeter equal to 4 ), while

$$
\widetilde{\mathcal{C}_{3}}=\{123,213,132\}, \quad \widetilde{\mathcal{C}}_{3}^{\prime}=\{321,312,231\} .
$$

However, $\zeta \in \widetilde{\mathcal{C}}_{n}$ does not imply that $\zeta^{M} \in \widetilde{\mathcal{C}}_{n}$. For instance, referring to the permutomino on the left of Figure 3 we have that $\pi_{1}^{M}=(5,2,4,3,1) \notin \widetilde{\mathcal{C}}_{5}$. More precisely we have that

$$
\widetilde{\mathcal{C}}_{n} \cap \widetilde{\mathcal{C}}_{n}^{\prime}=\left\{\Pi: \Pi \in \widetilde{\mathcal{C}}_{n} \text { and } \Pi^{M} \in \widetilde{\mathcal{C}}_{n}\right\}
$$

Thus, without loss of generality, we study the class $\widetilde{\mathcal{C}}_{n}$, i.e. we consider the problem of establishing if, for a given permutation $\Pi$ of $[n]=\{1, \ldots, n\}$, there is at least a convex permutomino $P$ of size $n$ such that $\pi_{1}(P)=\Pi$. Let us consider the sequence:

$$
i_{1}=1<i_{2}<\ldots<i_{h}<i_{h+1}<\ldots<i_{k}=n,
$$

with $1<i_{h} \leq n$, where $i_{h}=\Pi^{-1}(n)$, and

$$
\begin{aligned}
& i_{j-1}=\Pi^{-1}\left(\max \left\{\Pi\left(i_{j}-1\right), \Pi\left(i_{j}-2\right), \ldots, \Pi(1)\right\}\right), \quad j=h, \ldots, 2 \\
& i_{j+1}=\Pi^{-1}\left(\max \left\{\Pi\left(i_{j}+1\right), \Pi\left(i_{j}+2\right), \ldots, \Pi(n)\right\}\right), \quad j=h, \ldots, k-1 .
\end{aligned}
$$


Let us denote by $\mu$ the upper unimodal sequence:

$$
\Pi\left(i_{1}\right)=\Pi(1)<\Pi\left(i_{2}\right)<\ldots<\Pi\left(i_{h}\right)=n>\Pi\left(i_{h+1}\right)>\ldots>\Pi\left(i_{k}\right)=\Pi(n),
$$

and by $\sigma$ the sequence:

$$
\text { ( } \left.\Pi(1), \Pi\left(j_{1}\right), \ldots, \Pi\left(j_{r}\right), \Pi(n)\right),
$$

where $j_{1}, \ldots, j_{r}$ are the indices of $[n] \backslash \bigcup_{s=1}^{k} i_{s}$, with $1<j_{1}<\ldots<j_{r}<n$.

Example 1 Let us consider the convex permutomino of size 16 represented in Fig. 4 . We have

$$
\pi_{1}=(8,6,1,9,11,14,2,16,15,13,12,10,7,3,5,4),
$$

and we can determine:

i. the upper unimodal sequence $\mu=(8,9,11,14,16,15,13,12,10,7,5,4)$;

ii. the sequence $\sigma=(8,6,1,2,3,4)$ which turns out to be lower unimodal. In fact the following characterization holds.

Theorem 1 Let $\Pi$ be a permutation of $[n]$, such that $\Pi(1)<n$ and $\Pi(n)>1$. Let us consider the following two cases:

1) $\Pi(1)<\Pi(n)$; we have that $\Pi \in \widetilde{\mathcal{C}_{n}}$ if and only if $\sigma$ is lower unimodal;

2) $\Pi(1)>\Pi(n)$; we have that $\Pi \in \widetilde{\mathcal{C}_{n}}$ if and only if $\sigma$ is lower unimodal and $i_{j}+\Pi\left(i_{j+1}\right) \geq n+1$, for all $i_{h} \leq i_{j}<n$.

(Sketch of proof.) Let us consider the following discrete points: $A=\left(\pi_{1}^{-1}(1), 1\right), B=\left(1, \pi_{1}(1)\right)$, $C=\left(\pi_{1}^{-1}(n), n\right)$, and $D=\left(n, \pi_{1}(n)\right)$, and the following paths (see Figure 5 :

- the "upper path", running from $B$ to $D$ in a clockwise orientation, obtained connecting the couples of consecutive points of $\mu$, say $\left(i_{\ell}, \mu\left(i_{\ell}\right)\right)$, and $\left(i_{\ell+1}, \mu\left(i_{\ell+1}\right)\right)$, by means of segments $1^{\mu\left(i_{\ell+1}\right)-\mu\left(i_{\ell}\right)} 0^{i_{\ell+1}-i_{\ell}}$, where as usual 1 and 0 denote the vertical and horizontal unit steps, respectively.

- the "lower path" running from $B$ to $D$ in a counterclockwise orientation, obtained in an analogous way by connecting the points $(i, \sigma(i))$.

Let $P(\Pi)$ be the polygon obtained by connecting the upper and the lower paths. If we want $P(\Pi)$ to be a convex polygon, necessarily $\sigma$ must be lower unimodal. However this is not a sufficient condition for $P(\Pi)$ to be a polyomino, since the two paths may cross, as depicted in Figure 5 (b). We point out that, by construction, this possibility may occur only if $\Pi(1)>\Pi(n)$; more precisely, we have that the paths $B C$ and $D A$ can never cross, while the paths $C D$ and $A B$ cross if and only if the path $C D$ passes below the diagonal of the square parallel to the line $y=-x$, i.e. there is at least one index $s, h \leq s<k$, such that $i_{s}+\Pi\left(i_{s+1}\right)<n+1$.

Actually, as we investigate in the next section, there may be more than one convex permutomino associated with the permutation $\Pi$. It is not difficult to prove that the boundaries of these convex polyominoes coincide at least for the paths $C D$ and $A B$, thus if the two paths cross in $P(\Pi)$ they will cross in all the convex polygons associated with $\Pi$.

Example 2 Let us consider the permutation $\Pi=(4,8,1,9,7,3,5,2,6)$, with $\Pi(1)=4<\Pi(9)=6$; we have $\mu=(4,8,9,7,6)$, and $\sigma=(4,1,3,5,2,6)$. Since $\sigma$ is not lower unimodal, there is no convex permutomino $P$ such that $\Pi=\pi_{1}(P)$. However, as depicted in Figure 5 (a), there exists a non convex permutomino associated with $\Pi$.

Example 3 Let us consider the permutation $\Pi=(5,9,8,7,6,3,1,2,4)$, where $\mu=(5,9,8,7,6,4)$, and $\sigma=(5,3,1,2,4)$. Here $\sigma$ is lower unimodal, but being $\Pi(1)=5>\Pi(9)=4$ (case 2) of Theorem 1 ) this condition is not sufficient to give a convex permutomino; in fact, $i_{h}=\Pi^{-1}(9)=2$

$$
\begin{array}{l|lllll}
i_{j} & 2 & 3 & 4 & 5 & 9 \\
\hline \Pi\left(i_{j}\right) & 9 & 8 & 7 & 6 & 4
\end{array}
$$




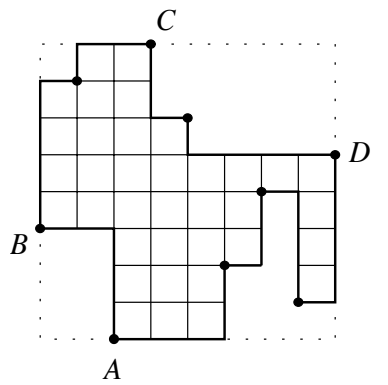

(a)

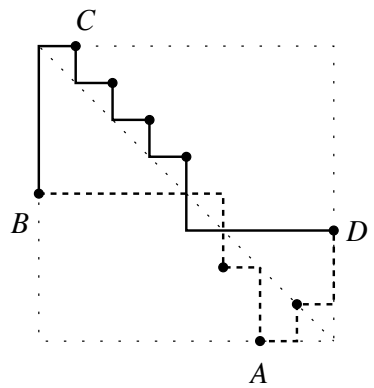

(b)

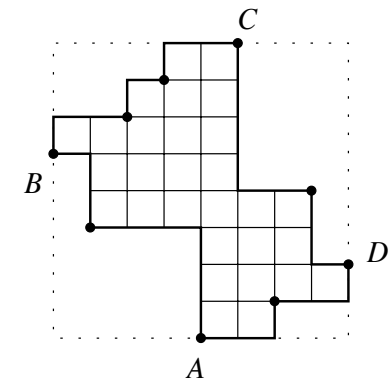

(c)

Fig. 5: (a) a non convex permutomino associated with $(4,8,1,9,7,3,5,2,6)$; (b) there is no convex permutomino associated with $(5,9,8,7,6,3,1,2,4)$; the "upper" and the "lower" paths have been represented by different lines; (c) the unique convex permutomino associated with $(6,4,7,8,1,9,2,5,3)$.

and $5+\Pi(9)=9<10$, hence there is no convex permutomino $P$ such that $\Pi=\pi_{1}(P)$, as depicted in Figure 5 (b).

Example 4 Let us consider the permutation $\Pi=(6,4,7,8,1,9,2,5,3)$ of $[9]$, where $\Pi(1)=6>\Pi(9)=$ 3 . Here we have $\mu=(6,7,8,9,5,3)$ and $\sigma=(6,4,1,2,3)$. Moreover, since $i_{h}=\Pi^{-1}(9)=6$, and

\begin{tabular}{l|lll}
$i_{j}$ & 6 & 8 & 9 \\
\hline$\Pi\left(i_{j}\right)$ & 9 & 5 & 3
\end{tabular}

we have $6+\Pi(8)=11 \geq 10$, and $8+\Pi(9)=11 \geq 10$, hence also the second requirement is satisfied and there is a convex permutomino associated with $\Pi$ (see Figure 5 (c)).

\section{Enumeration of some classes of convex permutominoes}

Let $P, P^{\prime} \in \mathcal{C}_{n}$. We say that $P \sim P^{\prime}$ if $\pi_{1}(P)=\pi_{1}\left(P^{\prime}\right)$. Obviously, $\sim$ is an equivalence relation on $\mathcal{C}_{n}$, and for any $P \in \mathcal{C}_{n}$ we may consider the equivalence class

$$
[P]_{\sim}=\left\{P^{\prime} \in \mathcal{C}_{n}: P^{\prime} \sim P\right\}
$$

i.e. the class of convex permutominoes associated with the permutation $\pi_{1}(P)$; from basic algebraic notions we have that the set $\mathcal{C}_{n / \sim}=\left\{[P]_{\sim}: P \in \mathcal{C}_{n}\right\}$ is in bijection with $\widetilde{\mathcal{C}}_{n}=\left\{\pi_{1}(P): P \in \mathcal{C}_{n}\right\}$. Thus, by abuse of notation we sometimes write $\left[\pi_{1}(P)\right]_{\sim}$ to mean $[P]_{\sim}$.

Lemma 1 Let $P \in \mathcal{D}_{n}$ (resp. $\left.\mathcal{P}_{n}\right)$; then $[P]_{\sim} \subseteq \mathcal{D}_{n}$ (resp. $\left.\mathcal{P}_{n}\right)$.

Lemma 1 says that all the convex permutominoes which are in $\sim$ relation with a directed-convex (parallelogram) permutominoes are still directed-convex (parallelogram).

We can now refine the statement of Theorem 1, and give a characterization of the permutations associated with some special classes of convex polyominoes. Let us consider the classes $\mathcal{D}_{n}, \mathcal{P}_{n}$, and $\mathcal{S}_{n}$ of directedconvex, parallelogram, and stack permutominoes of size $n$, and the associated classes of permutations, $\widetilde{\mathcal{P}}_{n}$, $\widetilde{\mathcal{D}}_{n}$, and $\widetilde{\mathcal{S}}_{n}$, respectively.

Theorem 2 Let $\Pi$ be a permutation of $[n]$, with $\Pi(1)<n$ and $\Pi(n)>1$. Then:

1. $\Pi \in \widetilde{\mathcal{D}}_{n}$ iff $\sigma$ is increasing (which implies $\Pi(1)=1$ );

2. $\Pi \in \widetilde{\mathcal{P}}_{n}$ iff $\mu$ and $\sigma$ are both increasing (which implies $\Pi(1)=1$ and $\Pi(n)=n$ );

3. $\Pi \in \widetilde{\mathcal{S}}_{n}$ iff $\sigma=(1, \Pi(n))$ (i.e. $\Pi(1)=1$ and $\Pi=\mu$ ).

The reader can verify the statement of Theorem 2 in the case of the polyominoes depicted in Figure 6

Now investigate the problem of determining how many elements are contained in the class $[P]_{\sim}$, for any $P \in \mathcal{C}_{n}$. 
(a)

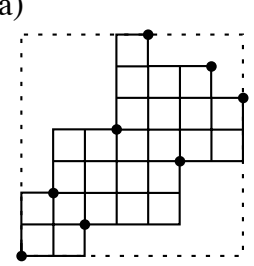

$\pi_{1}=(1,3,2,5,8,4,7,6)$

$\mu=(1,3,5,6,7,6)$

$\sigma=(1,2,4,6)$ (b)

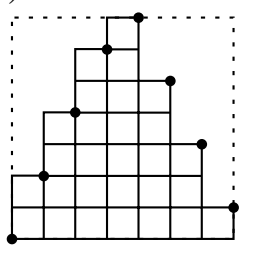

$\pi_{1}=\mu=(1,3,5,7,8,6,4,2)$

$\sigma=(1,2)$ (c)

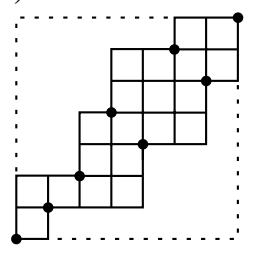

$\pi_{1}=(1,2,3,5,4,7,6,8)$

$\mu=(1,2,3,5,7,8)$

$\sigma=(1,4,6,8)$

Fig. 6: (a) a directed-convex permutomino; (b) a stack permutomino; (c) a parallelogram permutomino.

Theorem 3 Let $P \in \mathcal{C}_{n}$, and let $\mathcal{F}\left(\pi_{1}\right)$ be the subset of fixed points of $\pi_{1}$ in $\left\{\pi_{1}(1), \pi_{1}\left(i_{2}\right), \ldots, \pi\left(i_{h}\right)\right\}$, (i.e., the ascending part of the sequence $\mu$ ) which are different from 1 and $n$. Then we have:

$$
\left|[P]_{\sim}\right|=2^{\left|\mathcal{F}\left(\pi_{1}\right)\right|} .
$$

(Sketch of proof.) Let $P \in \mathcal{C}_{n}$, and let us consider the usual points $A, B, C$, and $D$ on the boundary of $P$. We observe that each fixed point of $\mathcal{F}\left(\pi_{1}\right)$ can belong to the "upper path" from $B$ to $C$ or to the "lower path" from $A$ to $D$; instead, due to the convexity constrain, the paths from $A$ to $B$, and from $C$ to $D$ are uniquely determined, and are equal for all the permutominoes of $[P]_{\sim}$. Hence there are $2^{|\mathcal{F}(P)|}$ possible convex permutominoes which can be obtained from $\pi_{1}$.

We remark that if $\pi_{1}(1)>\pi_{1}(n)$ then necessarily $\mathcal{F}\left(\pi_{1}\right)=\emptyset$, hence $\left|[P]_{\sim}\right|=1$.

Example 5 Let $P$ be the convex polyomino depicted in Fig. 7. We have that $\pi_{1}=(2,1,3,4,7,6,5)$, $\mu_{1}=(2, \mathbf{3}, \mathbf{4}, 7,6,5) \sigma_{1}=(2,1,5)$. The fixed points of $\pi_{1}$ are $3,4,6$, hence $\mathcal{F}\left(\pi_{1}\right)=\{3,4\}$, and finally by Theorem 3 we have that $\left|[P]_{\sim}\right|=2^{2}=4$. The four permutominoes associated with $\pi_{1}$ are depicted in Figure 7 The reader can try to find all the non convex permutominoes associated with $\pi_{1}$.
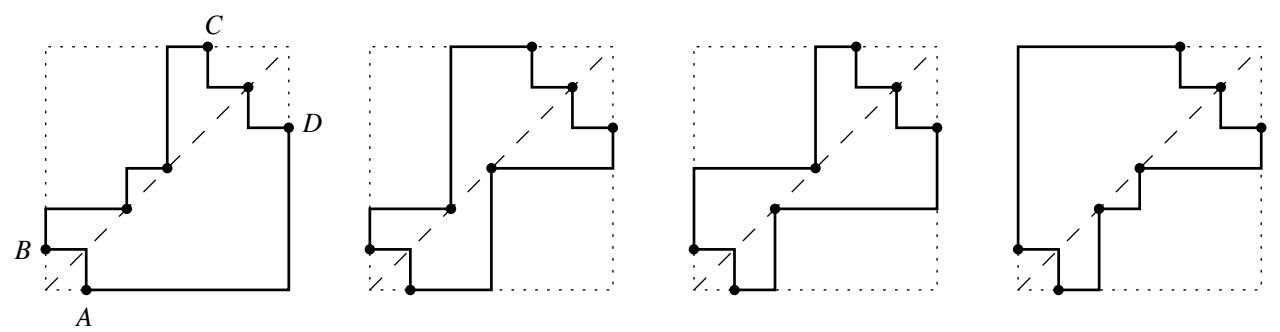

Fig. 7: The four convex permutominoes associated with the permutation $(2,1,3,4,7,6,5)$. Observe that the class is closed for symmetry through the diagonal $x=y$.

Now we provide a solution for the enumeration problems concerning the classes of parallelogram, directed-convex and stack polyominoes, and the associated classes of permutations.

\subsection{Parallelogram permutominoes}

Let us start by considering the class of parallelogram permutominoes.

Proposition 1 For any $n \geq 2$ we have:
i. $\left|\mathcal{P}_{n}\right|=c_{n-1}$;
ii. $\left|\widetilde{\mathcal{P}}_{n}\right|=c_{n-2}$. 
(Sketch of proof.) i) If a permutomino is parallelogram of size $n \geq 2$, its upper path must remain weakly above the main diagonal, i.e. it is a Dyck path of length $2(n-1)$, as shown in Figure 8 . Conversely, each Dyck path of length $2 n$ is the upper path of exactly one parallelogram permutomino of size $n+1, n \geq 1$; hence we have that $\left|\mathcal{P}_{n}\right|$ is given by the number of Dyck paths of length $2(n-1)$, which is equal to $c_{n-1}$.
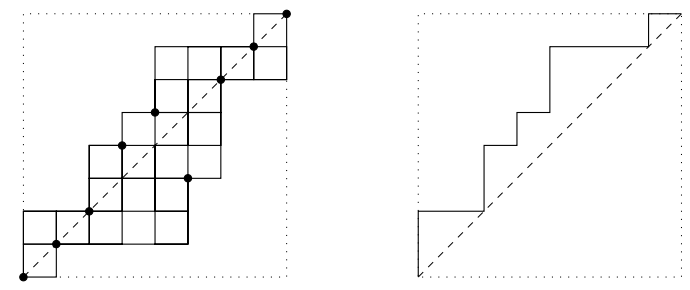

Fig. 8: A parallelogram permutomino (associated with the permutation $\pi_{1}=(1,2,3,5,6,4,7,8,9)$ ) and its associated Dyck path.

ii) Let $\Pi$ be a permutation of $\widetilde{\mathcal{P}}_{n}, n \geq 2$. We agree to represent $\Pi$ by the unique parallelogram permutomino of $[\Pi] \sim$ for which the upper path is an elevated Dyck path (i.e. the permutomino such that all the fixed points in $\Pi$ belong to the lower path except 1 and $n$ ). Let $w(\Pi)$ be the upper path of such a permutomino from which we have removed the first and last step (a vertical and an horizontal one, respectively). Necessarily $w(\Pi)$ is a Dyck path of length $2(n-2)$ (see Figure 9).

Conversely, given any Dyck path $w$ of length $2(n-2)$, with $n \geq 2$, we elevate it by adding an initial vertical step and a final horizontal step, and build up the unique parallelogram permutomino $P(w)$ having such obtained path as upper path. Then we have that $\pi_{1}(P(w)) \in \widetilde{\mathcal{P}}_{n}$. As a consequence, the number of permutations of $\widetilde{\mathcal{P}}_{n}$ is equal to the number of Dyck paths of length $2(n-2)$, i.e. $c_{n-2}$.
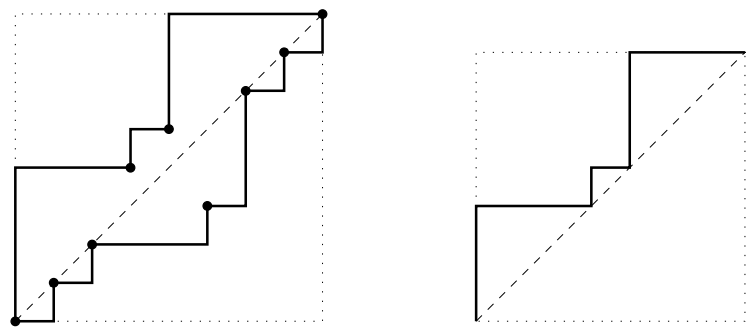

Fig. 9: A parallelogram permutomino representing the permutation $\pi_{1}=(1,2,3,5,6,4,7,8,9)$ and the associated Dyck path.

\subsection{Directed-convex permutominoes}

In order to study the class of directed-convex permutominoes we use the same approach we have used for parallelogram permutominoes. On a directed-convex permutominoes we identify the following points: $O=(1,1), C=\left(i_{h}, n\right), D=\left(n, \pi_{1}(n)\right)$ (see Fig. 10 ). Moreover, let $X=\left(x, \pi_{1}(n)\right), x \leq \pi_{1}(n)$, be the point obtained from the intersection of the path from $O$ to $C$ with the line $y=\pi_{1}(n)$.

Trivially, a directed-convex permutomino $P$ is uniquely determined by its "upper path", i.e. the path that goes from $O$ to $D$ in a clockwise orientation. We encode such a path (hence the permutomino $P$ ) in terms of a path, called $\phi(P)$, running from $(1,1)$ to $(n, n)$, where the sequences of consecutive horizontal steps can be of two different types, say solid or dotted. More precisely, we associate with $P$ a path $\phi(P)$ obtained by concatenating:

- the path from $O$ to $X$,

- a path obtained by projecting over the path from $X$ to $C$ all the horizontal steps of the path from $C$ to $D$ (such projected horizontal steps are denoted by means of dotted steps, as shown in Fig. 10.

Lemma 2 A directed-convex (non parallelogram) permutomino $P$ of size $n$ is encoded by a 2-colored Dyck path $\phi(P)$ of length $2(n-1)$ running from $(1,1)$ to $(n, n)$, which can be uniquely decomposed into two parts: 


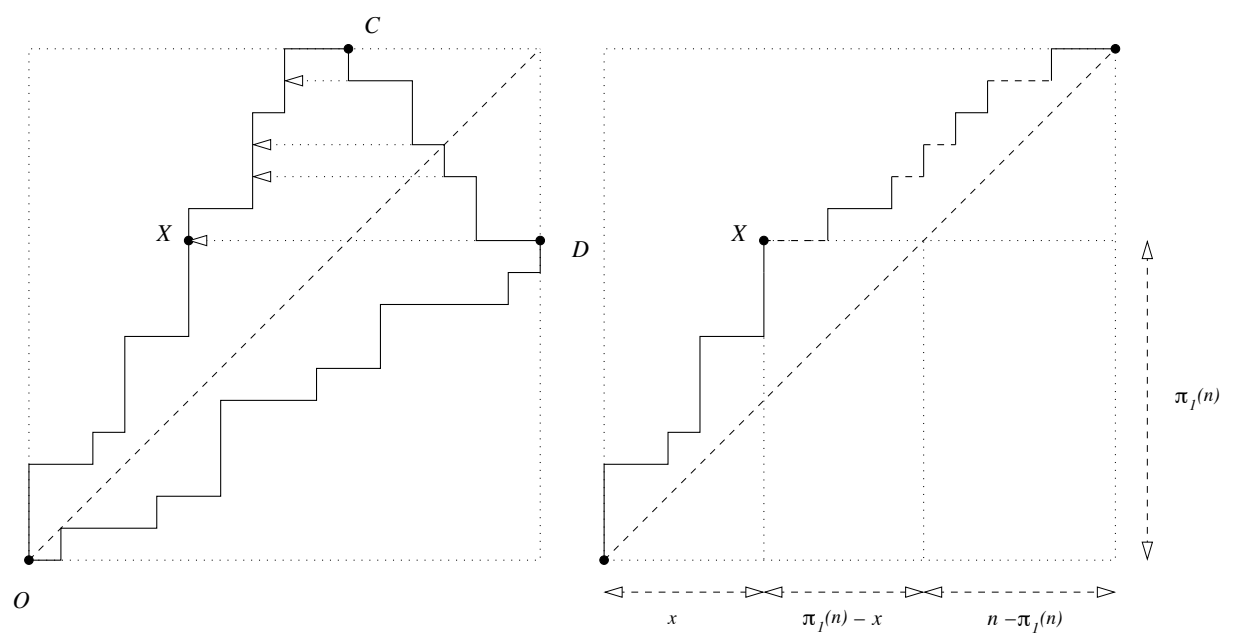

Fig. 10: A directed-convex permutomino and its associated two-colored Dyck path.

1. a non-empty path of the form: $1^{+}\left(0^{+} 1^{+}\right)^{*}$;

2. a non-empty path of the form: $\overline{0}^{+} 1\left(0^{+} 1 \vee \overline{0}^{+} 1\right)^{*} 0^{+}$,

where $0, \overline{0}$, and 1 denote respectively the solid horizontal, dotted horizontal, and vertical unit steps.

Vice versa it can be easily proved that each 2-colored Dyck path from $(1,1)$ to $(n, n)$ which can be decomposed into two parts satisfying properties 1 . and 2. leads to a unique directed-convex (non-parallelogram) permutomino.

Proposition 2 For any $n \geq 2$ we have that $\left|\mathcal{D}_{n}\right|=\frac{1}{2} b_{n-1}$.

(Sketch of proof.) It is possible to determine a bijection between the class of directed-convex permutominoes of size $n \geq 2$ and Grand-Dyck paths having length equal to $2(n-1)$ and ending with an horizontal step. We recall the a Grand-Dyck path of length $2 n$ is simply a path from $(0,0)$ to $(n, n)$ using vertical and horizontal unit steps. Let $P$ be a directed-convex permutomino of size $n$ :

1. if $P$ is a parallelogram one, then it is associated with a Dyck path of length $2(n-1)$ (Proposition 1 ;

2. otherwise, using the encoding of Lemma 2, $P$ can be mapped into a (non Dyck) Grand-Dyck path having length equal to $2(n-1)$ and ending with an horizontal step.

Finally, $\left|\mathcal{D}_{n}\right|$ is given by the number of Grand-Dyck paths ending with an horizontal step, i.e. $\frac{1}{2} b_{n-1}$.

Proposition 3 For any $n \geq 2$ we have that $\left|\widetilde{\mathcal{D}}_{n}\right|=b_{n-2}$.

\subsection{Stack permutominoes}

The case of stack permutominoes is slightly different from the previous cases, since the statement of Lemma 1]does not hold for this class. We have indeed the following:

Proposition 4 Let $\Pi$ be an upper unimodal permutation of size $n$, with $\Pi(1)=1, \Pi=\mu$; then $[\Pi]_{\sim} \subseteq \mathcal{D}_{n}$, and there is exactly one stack polyomino associated with $\Pi$, i.e. $\left|[\Pi]_{\sim} \cap \mathcal{S}_{n}\right|=1$.

Proposition 5 For any $n \geq 2$ we have that $\left|\mathcal{S}_{n}\right|=2^{n-2}$.

(Sketch of proof.) A stack permutaomino $P$ of size $n \geq 2$ is uniquely determined by its associated permutation $\pi_{1}(P)$, which is upper unimodal. The number of such permutations with the number $n$ in the $k$ th position, $1<k \leq n$, is clearly given by $\left(\begin{array}{l}n-2 \\ k-2\end{array}\right)$, hence follows the thesis.

Example 6 Let us consider $\Pi=(1,2,3,5,4)$; we have $\Pi(1)=1$ and $\mu=\Pi$. Hence, according to Theorem 2 and Lemma 1 we have that $[\Pi]_{\sim} \subseteq \mathcal{D}_{n}$, and from Theorem 3 we have that $\left|[\Pi]_{\sim}\right|=4$ (see Figure 11). But Proposition 4 ensures that only one of the four directed-convex permutominoes associated with $\Pi$ is a stack (precisely, the leftmost in Figure 11 ). 

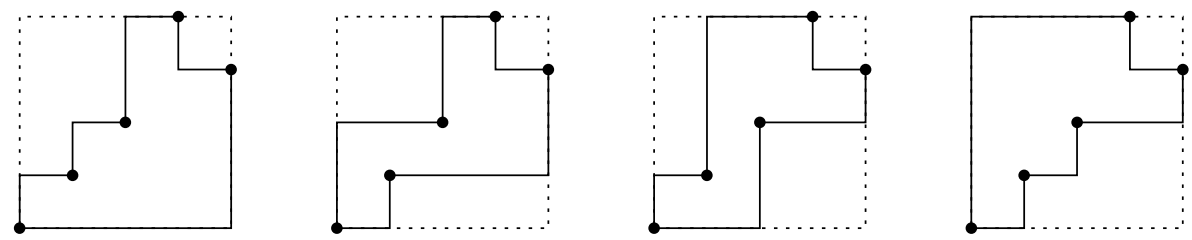

Fig. 11: The four directed-convex polyominoes associated with $\Pi=(1,2,3,5,4)$.

\section{Open problems}

In this paper we have considered the class of convex permutominoes; the main results are the characterization of the set of permutations associated with convex permutominoes, and the enumeration of some classes of convex permutominoes, including the stack, parallelogram, and the directed-convex ones. There are however several problems concerning permutominoes which are still open. Regarding convex permutominoes, the major open problems are the following:

1. determine the number of convex permutominoes, $\left|\mathcal{C}_{n}\right|$,

2. determine the number of permutations associated with convex permutominoes, $\left|\widetilde{\mathcal{C}_{n}}\right|$, and $\left|\widetilde{\mathcal{C}}_{n} \cap \widetilde{\mathcal{C}}_{n}^{\prime}\right|$,

3. determine the cardinalities of the two subsets of $\widetilde{\mathcal{C}}_{n}$,

$$
\widetilde{\mathcal{C}_{n}^{1}}=\left\{\Pi \in \widetilde{\mathcal{C}_{n}}: \Pi(1)<\Pi(n)\right\}, \quad \widetilde{\mathcal{C}_{n}^{2}}=\widetilde{\mathcal{C}_{n}} \backslash \widetilde{\mathcal{C}_{n}^{1}}
$$

Trivially, for any $n \geq 2$, we have $\widetilde{\mathcal{C}_{n}^{1}}>\widetilde{\mathcal{C}_{n}^{2}}$.

4. determine the number of convex permutominoes having area $m \geq 1$.

Concerning the whole class of permutominoes, our goal is to generalize some of the properties stated for convex polyominoes. In particular, we wish to give a characterization of the set of permutations associated with permutominoes, and then of the set $[P]_{\sim}=\left\{P^{\prime}\right.$ is a permutomino : $\left.P^{\prime} \sim P\right\}$.

Acknowledgments. The authors wish to thank F. Incitti for suggesting the problem of enumerating the class of permutominoes. Moreover special thanks are due to G. Droandi and S. Socci (University of Siena) who wrote a program for the exhaustive generation of permutominoes, which provided us the first terms of the studied series, allowing us to test conjectures and to double check our results.

\section{References}

[BMRR] G. Barequet, M. Moffie, A. RibÛ and G. Rote, Counting Polyominoes on Twisted Cylinders, Proceedings of 2005 European Conference on Combinatorics, Graph Theory and Applications (EuroComb '05), Ed. S. Felsner, Discrete Mathematics and Theoretical Computer Science Proceedings AE, pp. 369-374

[DV] M. Delest, X. Viennot, Algebraic languages and polyominoes enumeration, Theor. Comp. Sci., 34 169-206 (1984).

[I] F. Incitti, Permutation diagrams, fixed points and Kazdhan-Lusztig R-polynomials, Ann. Comb., (to appear).

[JG] I. Jensen, A. J. Guttmann, Statistics of lattice animals (polyominoes) and polygons, J. Phys. A:Math. Gen., 33, (2000), 257-263.

[S] R. P. Stanley, Enumerative Combinatorics, Vol.2, Cambridge University Press, Cambridge (1999). 
